\title{
Circulating Plasma miRNA-21 as a Superior Biomarker Compared to CA 15-3: Assessment in Healthy Age Matched Subjects and Different Stage of Breast Cancer Patients
}

\author{
Merlyna Savitri ${ }^{1,2,3}$, Ugroseno Yudho Bintoro ${ }^{1,2,3}$, Made Putra Sedana ${ }^{1,2,3}$, \\ Muhammad Noor Diansyah ${ }^{1,2,3}$, Pradana Zaky Romadhon ${ }^{1,2,3}$, Putu Niken Ayu Amrita ${ }^{1,2,3}$, \\ Andi Yasmin Wijaya ${ }^{3}$, Winona May Hendrata ${ }^{3}$, Ami Ashariati Prayogo 1,2,3, \\ ${ }^{1}$ Hematology-Medical Oncology Division, Department of Internal Medicine, Dr. Soetomo Teaching Hospital, Jl. Mayjen Prof. Dr. Moestopo \\ No.6-8, Surabaya, Indonesia \\ ${ }^{2}$ Hematology-Medical Oncology Division, Department of Internal Medicine, Universitas Airlangga Hospital, Jl. Mayjen Prof. Dr. Moestopo No.47, \\ Surabaya, Indonesia \\ ${ }^{3}$ Faculty of Medicine, Universitas Airlangga, J1. Mayjen Prof. Dr. Moestopo No.47, Surabaya, Indonesia \\ *Corresponding author. E-mail: amiashariati@yahoo.com
}

Received date: Jan 23, 2020; Revised date: May 1, 2020; Accepted date: May 4, 2020

\section{Abstract}

ACKGROUND: Carbohydrate antigens 15-3 (CA 15-3) is a conventional tumor marker in breast cancer, with low sensitivity and specificity. MicroRNA (miRNA)-21 showed its stability in circulation and could serve as powerful biomarker. The aim of this study was to evaluate miRNA-21 as breast cancer biomarker compared to CA 15-3 in Indonesian population.

METHODS: Circulating plasma miRNA-21 expression was measured using qRT-PCR in 49 patients at various stages of breast cancer and 16 healthy controls. The relative expression value of miRNA-21 was calculated using $2^{-\Delta \Delta \mathrm{Ct}}$. Meanwhile, CA 15-3 was quantified using electrochemiluminescence immunoassay (ECLIA) methods. The results of miRNA-21 and CA 15-3 plasma circulating expression were compared with controls at each stage and between stages of breast cancer.

RESULTS: CA 15-3 median level in breast cancer group was 1.60 times higher compared to control group $(p=0.019)$,

\section{Introduction}

World-widely, breast cancer is well known as the most prevalent malignancy in women, in line with its morbidity and mortality.(1) The high prevalence of breast cancer
$21.00 \mu / \mathrm{mL}$ and $13.05 \mu / \mathrm{mL}$, respectively. Median miRNA-21 expression in breast cancer group was elevated 4.92 folds compared to control group $(p=0.001), 4.43$ and 0.90 , respectively. There was no significant difference of CA 15-3 level between controls and all stages of breast cancer group. CA 15-3 cut-off value was $15.05 \mu / \mathrm{mL}(p=0.016)$ with $59.2 \%$ sensitivity and $62.5 \%$ specificity. Meanwhile, there was a significant difference of miRNA-21 expression between controls and most stages of breast cancer group. Circulating miRNA-21 expression cut-off value was 2.07 ( $p=0.000$ ) with $91.8 \%$ sensitivity and $87.5 \%$ specificity.

CONCLUSION: Circulating miRNA-21 expression and CA 15-3 levels were significantly increased in breast cancer group compared to control group. The miRNA-21 expression increased consistently with breast cancer stage progression. miRNA-21 could serve as superior biomarker compared to CA 15-3.

KEYWORDS: biomarker, breast cancer, circulating plasma, liquid biopsy, miRNA-21

Indones Biomed J. 2020; 12(2): 157-64 used to be dominated by the western countries. However, nowadays breast cancer incidence trends shifts towards developing countries and Asian population, including Indonesia.(2)

The management of breast cancer requires a comprehensive approach, consisting diagnostic, therapeutic 
and monitoring procedures to ensure better outcome in mortality and morbidity.(3) Being an initial stage in the management of breast cancer, the diagnostic procedure is a critical point, along with its prognosis.(3)

The present standardized triple test approach for breast cancer, which includes clinical, radiological and histopathological examinations, is well known for its decent specificity and sensitivity. However, there are several issues lies in this approach.(4,5) Meanwhile, mammography has shown a limitation in young women with a dense breast tissue.(6) Invasiveness of breast biopsy is also considered to be inconvenient for some patients.(7)

Non-invasive and minimal-invasive methods of diagnostic are also proved to be preferable modalities compared to an invasive procedure. There are several established tumor marker that are well known in routine practice, including carcino-embryonic antigen (CEA), carbohydrate antigens 15-3 (CA 15-3), and carbohydrate antigens 27-29 (CA 27-29).(8) Yet, these biomarkers show relatively poor sensitivity and specificity.(9) The issues of CA 15-3 detection especially caused by an increase of CA 15-3 levels in several particular diseases, including liver cirrhosis and other adenocarcinoma, which hinders its specificity. $(10,11)$ In the recent development, circulating breast cancer biomarker research is still performed worldwide in a quest to pinpoint the particular biomarker molecules.

miRNA is present in the circulation and known to be an important regulator molecules in cells metabolism and oncogenesis.(12) Moreover, miRNA also showed relatively stable nature. Existence of several constantly endogeneous expressed miRNA that can be utilized as a miRNA baseline might strengthen its potential as a biomarker in malignancy.(13) In breast cancer there are several miRNA that have proved to correlate with disease progression, either in line or inversely. The search continues to find the most suitable miRNA, which correlates with breast cancer progression. $(3,12)$ miRNA-21, miRNA-29b, and miRNA-155 have been shown to have an in line correlation to breast cancer progression.(14) However miRNA-21 shows relatively favorable correlation compared to other miRNAs.(14)

miRNA-21 is known as a regulator of several identified target genes, including tumor suppressor tropomyosin 1 (TPM1), network of p53, PDCD4, PTEN, and Maspin. (13) miRNA-21 interacts with these genes and regulated cells proliferation, oncogenesis, and cell migration.(15) Supported by the previous studies, miRNA-21 was also used for evaluation of breast cancer progression, prognosis, and survival.(16) Previous study also showed a correlation between circulating level of miRNA-21, circulating tumor cells and tissue level of miRNA-21 which indicated that measurement of circulating miRNA-21 level could potentially represent breast cancer progression.(14,17) Therefore, based on these data the investigation of miRNA-21 as a breast cancer biomarker for the disease's progressivity monitoring and diagnostic should be performed especially in Indonesia. The importancy of assessing miRNA level in healthy population is also needed, as the miRNA-21 evaluation in Indonesian population was never been assessed before. This data could suggest whether miRNA expression could serve as a breast cancer biomarker in Indonesian population.

\section{Methods}

\section{Subject Selection}

In this study, serum samples from 65 women above 18 years old were analyzed. Among them were 49 patients with breast cancer and 16 healthy women as control. Samples were taken from newly diagnosed breast cancer (stage 1-4) patients recruited from the Hematology and Medical Oncology Outpatient Clinic of Universitas Airlangga Hospital, Surabaya, before undergoing chemotherapy and radiotherapy. Breast cancer diagnosis was confirmed by physical examination, radiological examination, and histopathological examination. The samples of healthy control were obtained from age-matched women, who had no history of malignancy or comorbid disease such as chronic hepatitis, liver cirrhosis and has been proven to be healthy by physical examination.

Written informed consent from all patients was obtained for blood collection at the unit of clinical laboratory of Universitas Airlangga Hospital. This process was approved by Bioethical Committee of Universitas Airlangga Hospital (Ethical Clearance Document Registry Number 164/KEP/2019). The entire subject characteristics data were collected from patient medical record. Menarche status and other data that were not obtained in medical record was obtained from anamnesis.

Blood sample collection was performed to the entire subjects after receiving written informed consent in Hematology-Medical Oncology policlinic, Universitas Airlangga Hospital, The $10 \mathrm{ml}$ peripheral blood was collected through venipuncture. The blood then stored in ethylenediaminetetraacetic acid (EDTA) vacuum container and divided into two tubes for both CA 15-3 and miRNA-21 examination. 


\section{CA 15-3 Examination}

Plasma from peripheral venous blood was obtained after collection to EDTA containing vacuum container and incubation for 1 hour in room temperature. Before plasma was collected the tube were centrifuged 5 minutes in $1900 \mathrm{G}$ to ensure purity of plasma. A total of $20 \mu \mathrm{L}$ of subject plasma was diluted to 1:10 with Diluent Universal. After dilution the streptavidin-coated microparticle was added for sticking it to solid phase, forming a reaction mixture. Reaction mixture was transferred to measuring cell where the microparticles are captured by magnetic electrode, unbound substances then removed by washing with ProCell/ProCell M (Roche Diagnostics GmbH, Mannheim, Germany). Electrical current then applied to electrode that will producing a electroluminescent signal which will be captured by photomultiplier. Determination of electrochemiluminescent signal was performed by based on calibration curve from 2-point calibration and master curve, which present with each reagent barcode. The level of CA 15-3 was obtained from an automatic process of quantification through COBAS ECLIA analyzer (Roche Diagnostics, Basel, Switzerland). This procedure was performed according to Elecsys CA 15-3 II ECLIA kit. Normal reference limit that used was $\leq 25 \mu / \mathrm{mL}$. CA 15-3 examination was performed in the Prodia Clinical Laboratory, Surabaya and Jakarta.

\section{Blood Sample Preparation}

Plasma separation was performed by centrifuging EDTA venous blood sample from EDTA vacuum container after 1 hour storage at room temperature using a swinging bucket rotor for 10 minutes at $1900 \mathrm{G}$ and $4^{\circ} \mathrm{C}$. Plasma from the sample was collected without disturbing buffy coat layer. Collected plasma then transferred to conical tubes and recentrifuged for 10 minutes at $16000 \mathrm{~g}$ and $4{ }^{\circ} \mathrm{C}$ in a fixedangle rotor. Clear plasma supernatant was collected without disturbing the pellet.

\section{RNA Extraction and Reverse Transcription}

Total RNA extraction was performed following manufacturer's instruction of the Qiagen miRNeasy Serum/ Plasma Kit (Cat. No. 217184, Qiagen, Hilden, Germany). The quantification of total RNA was performed using NanoDrop $^{\text {TM }}$ (Thermo Scientific, Massachuset, USA).

Total isolated RNA from plasma then was transferred and underwent reverse transcription for producing cDNA that needed for qRT-PCR analysis. Reverse transcription was carried out using TaqMan MicroRNA Reverse Transcription kit (Cat. No. 4366596, Applied Biosystems,
Carlsbad, California, USA) by mixing $3 \mu \mathrm{L}$ of RT primer and $5 \mu \mathrm{L}$ RNA sample (contains 1-10ng RNA) with $7 \mu \mathrm{L}$ of reverse transcription reaction mix. Thermal cycle setting that used was $16^{\circ} \mathrm{C}$ for 30 minutes, $42^{\circ} \mathrm{C}$ for 30 minutes, $85^{\circ} \mathrm{C}$ for 5 minutes and $4^{\circ} \mathrm{C}$ for holding reaction. The primer that was utilized are Hsa-miR-21-3p (miRNA-21) and HsamiR-16-5p (miRNA-16) as endogenous control.

\section{Quantitative Real-Time Polymerase Chain Reaction (qRT-PCR)}

qRT-PCR from cDNA was performed using TaqMan MicroRNA assay (Applied Biosystems), TaqMan endogenous control assay for miRNA-16 (Applied Biosystems), and Taqman_Universal PCR master mix II, No UNG (Applied Biosystems) by transferring and mixed previous cDNA with PCR Reaction mix. The thermal cycle setting for qRT-PCR was $95^{\circ} \mathrm{C}$ for 10 minutes $(1$ cycle), $95^{\circ} \mathrm{C}$ for 15 seconds, and $60^{\circ} \mathrm{C}$ for 60 seconds ( 40 cycles).

\section{Data Analysis}

The data that was acquired which encompass patient characteristics, CA-15-3 level, and circulating miRNA-21 expression. The data analysis were carried out by performing homogeneity test for age-matching and menarche status-matching using Chi-square test, normality distribution analysis (Levene's Test) and non-parametric statistic analysis by using IBM SPSS Statistics v.24.0 (IBM Corporation, New York, USA).

\section{Results}

\section{Demographic Characteristics}

Age matching analysis was performed to represent the healthy control groups as a comparison to breast cancer subjects groups for minimizing the relative risk of breast cancer based on the age variables. Menarche status between healthy control subjects and breast cancer subjects group also has been matched for minimizing the breast cancer hormonal relative risk difference in pre-menopause and menopause subjects. In the breast cancer group $(n=49)$, the research subjects with stage $1(n=5)$ breast cancer were $10.2 \%$, stage 2 was $42.85 \%(\mathrm{n}=21)$, stage 3 was $20.40 \%$ $(\mathrm{n}=10)$ subjects, and stage 4 in this study was found in $26.53 \%(\mathrm{n}=13)$ subjects (Table. 1$)$

\section{Circulating miRNA-21 Level Expression}

The circulating miRNA-21 level expression was different in breast cancer and healthy control subjects. The expression 
Table 1. Demographic characteristic.

\begin{tabular}{|c|c|c|c|}
\hline \multirow[b]{2}{*}{ Variable } & \multicolumn{2}{|c|}{ n (\%) } & \multirow[b]{2}{*}{$p$-value } \\
\hline & $\begin{array}{l}\text { Breast Cancer Subjects } \\
\qquad(n=49)\end{array}$ & $\begin{array}{l}\text { Healthy Control Subjects } \\
\qquad(\mathrm{n}=16)\end{array}$ & \\
\hline Age (years), mean \pm SD & $51.95 \pm 10.21$ & $49.75 \pm 10.42$ & 0.925 \\
\hline Menarche status & & & 0.940 \\
\hline Pre-menopause & $25(51.02)$ & $9(56.25)$ & \\
\hline Menopause & $24(48.97)$ & $7(43.75)$ & \\
\hline \multicolumn{4}{|l|}{ Tumor staging } \\
\hline Stage 1 & $5(10.20)$ & & \\
\hline Stage 2 & $21(42.85)$ & & \\
\hline Stage 3 & $10(20.40)$ & & \\
\hline Stage 4 & $13(26.53)$ & & \\
\hline
\end{tabular}

Age matching and menarche status matching in the breast cancer groups and healthy subjects group (Mann-Whitney test) with breast cancer patient cancer staging.

of miRNA-21 in the breast cancer group as a whole was 4.92 times higher than the expression of miRNA-21 in the control group. A significant difference was obtained in the comparison of the expression of circulating miRNA-2 breast cancer groups at among stage I, II, III, IV breast cancer patient, and control group $(p=0.001)$ (Table 2).

\section{Circulating miRNA-21 Expression Level and CA 15-3 Level Sensitivity and Specificity}

The cut-off value of CA 15-3 was obtained in the breast cancer group of 15.05 with an area under the ROC curve (ROC-AUC) of $0.701(70.1 \%$, with $p=0.016)$ and sensitivity of $59.2 \%$ and specificity by $62.5 \%$.
On the other hand, the expression of circulating miRNA-21 in the breast cancer group, showed a cut-off value of 2.07 with an area under the ROC curve (ROCAUC) of 0.931 (93.1\%, with $p=0.000)$, and a sensitivity of $91.8 \%$ and specificity of $87.5 \%$ (Figure 1 ).

\section{CA 15-3 Level Expression}

In this study, CA 15-3 levels in the breast cancer group were 1.60 times higher compared to CA 15-3 levels in the control group. The results obtained were significant differences in the comparison of CA levels of 15-3 breast cancer groups at among stage $1,2,3,4$, and control groups $(p=0.019)$ (Table 3).

Table 2. Circulating miRNA-21 level expression comparison in different stage of breast cancer and healthy control subjects.

\begin{tabular}{|c|c|c|c|c|}
\hline \multirow{2}{*}{ Variable } & \multicolumn{3}{|c|}{ Circulating miRNA-21 Expression $\left(2^{-\Delta \Delta C t}\right)$} & \multirow{2}{*}{$p$-value } \\
\hline & Median (Min-Max) & Mean \pm SD & $\begin{array}{c}\text { Median Fold } \\
\text { Change }\end{array}$ & \\
\hline Healthy control subjects $(n=16)$ & $0.90(0.16-4.53)$ & $1.35 \pm 1.18$ & 4.92 & 0.001 \\
\hline Breast cancer subjects $(n=49)$ & $4.43(1.11-32.22)$ & $6.00 \pm 5.35$ & & \\
\hline Stage $1 \quad(n=5)$ & $4.75(2.88-5.35)$ & $4.31 \pm 0.97$ & & \\
\hline Stage $2(n=21)$ & $3.68(1.18-11.79)$ & $4.62 \pm 3.13$ & & \\
\hline Stage $3 \quad(n=10)$ & $3.83(1.11-14.12)$ & $5.40 \pm 4.27$ & & \\
\hline Stage $4(n=13)$ & $5.77(3.18-32.22)$ & $9.32 \pm 8.21$ & & \\
\hline Early stage breast cancer (Stage $1 \& 2$ ) & $3.93(1.18-11.79)$ & & & \\
\hline Advanced stage breast cancer (Stage $3 \&$ \&) & $5.09(1.11-32.22)$ & & & \\
\hline
\end{tabular}




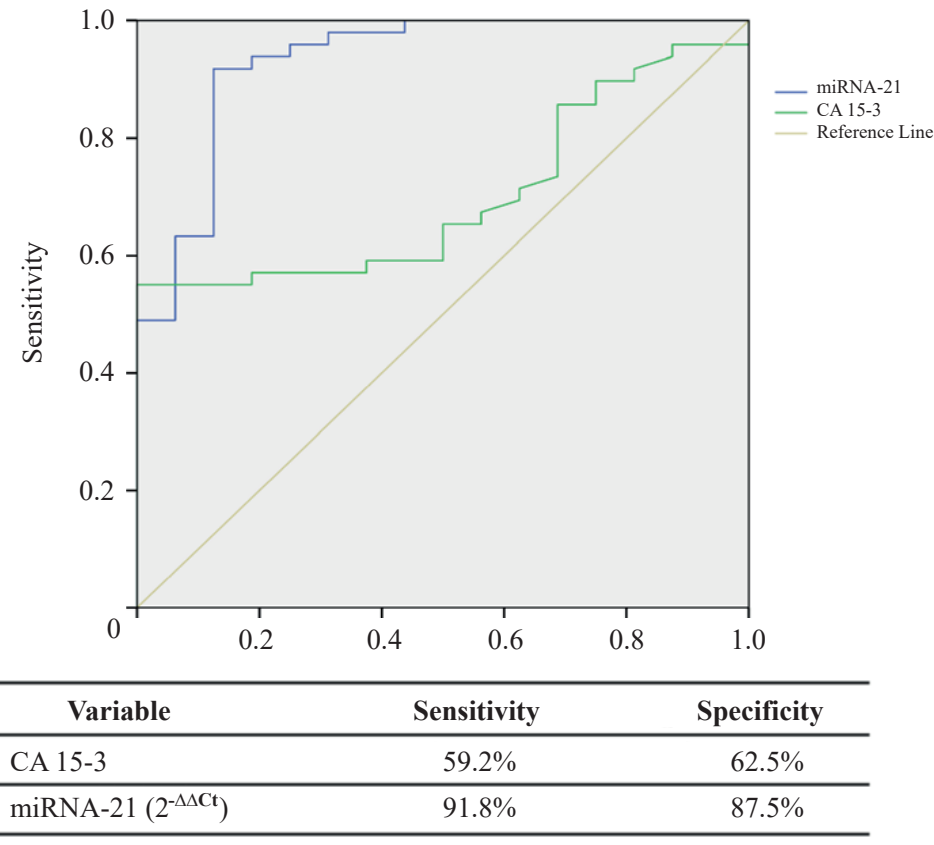

Figure 1. ROC curve of CA 15-3 and miRNA-21 in distinguishing between breast cancer and healthy subjects.

\section{Comparison of Circulating miRNA-21 Level and CA}

\section{5-3 Level}

In this study, CA levels 15-3 had no meaningful results in the comparison between controls with all stages of breast cancer and the comparison between stages in the breast cancer group. The miRNA-21 level in control group was significantly different compared to subjects in breast cancer group with stage 1, 2, and 4. However, there was no significant difference between control group and subject with stage 3 breast cancer (Table 4, Figure 2).

\section{Discussion}

This study was performed for assessing the clinical utility of circulating miRNA-21 expression measurement in patients with breast cancer in comparison to CA 15-3 as a conventional breast cancer marker.(12,18,19) In this research, venous blood plasma sample as source material for circulating plasma miRNA-21 expression measurement was chosen due to its advantage over serum materials. Occurrence of hemolysis is more minimal during plasma collection compared to serum which minimized confounding factors for miRNA-21 expression analysis.(20)

CA 15-3, a form of surface-soluble protein-linked Mucin 1 (MUC-1), including the Mucin family. It is also known as MUC-1 and is involved in cell protection and lubrication. Mucin 1 is a high molecular weight $(300 \mathrm{KDa})$ glycoprotein. CA 15-3 is expressed in the apical aspects of the glandular epithelium and breast cell ducts. CA 15-3 measurement is useful as a marker for detecting recurrence and monitoring the treatment of metastatic breast cancer.(4)

Table 3. CA 15-3 level expression comparison in different stage of breast cancer and healthy control subjects.

\begin{tabular}{|c|c|c|c|c|}
\hline \multirow[b]{2}{*}{ Variable } & \multicolumn{3}{|c|}{ CA $15-3(\mu / \mathrm{mL})$} & \multirow[b]{2}{*}{$p$-value } \\
\hline & Median (Min-Max) & $\operatorname{Mean} \pm$ SD & $\begin{array}{c}\text { Median Fold } \\
\text { Change }\end{array}$ & \\
\hline Healthy control subjects $(n=16)$ & $13.05(5.2-19.9)$ & $12.65 \pm 5.13$ & 1.6 & 0.019 \\
\hline Breast cancer subjects $(n=49)$ & $21.00(4.73-3000)$ & $128.27 \pm 441.74$ & & \\
\hline Stage $1 \quad(n=5)$ & $9.80(4.73-27.8)$ & $12.24 \pm 9.25$ & & \\
\hline Stage $2(n=21)$ & $12.40(6.30-57.2)$ & $18.48 \pm 13.83$ & & \\
\hline Stage $3 \quad(n=10)$ & $28.30(8.94-130.20)$ & $37.06 \pm 34.68$ & & \\
\hline Stage $4(n=13)$ & $98.50(5.00-3000)$ & $420.40 \pm 808.21$ & & \\
\hline
\end{tabular}

CA 15-3 level in the breast cancer group was significantly different compared to healthy groups with 1.6 fold median changes (Mann-Whitney test). 
Table 4. Comparison of miRNA-21 and CA 15-3 in distinguishing different stage of breast cancer and control.

\begin{tabular}{ccc}
\hline \multirow{2}{*}{ Stage } & \multicolumn{2}{c}{$p$-value } \\
\cline { 2 - 3 } & CA 15-3 & miRNA-21 \\
\hline Control/Stage 1 & 1.000 & 0.003 \\
Control/Stage 2 & 0.407 & 0.001 \\
Control/Stage 3 & 0.256 & 0.089 \\
Control/Stage 4 & 0.407 & 0.03 \\
Stage 1/Stage 2 & 0.743 & 0.995 \\
Stage 1/Stage 3 & 0.278 & 0.936 \\
Stage 1/Stage 4 & 0.406 & 0.255 \\
Stage 2/Stage 3 & 0.51 & 0.984 \\
Stage 2/Stage 4 & 0.42 & 0.325 \\
Stage 3/ Stage 4 & 0.465 & 0.587 \\
\hline
\end{tabular}

Unfortunalety, CA 15-3 sensitivity and specificity are low at $22.4 \%$ and $15.7 \%$ respectively for detecting early breast cancer.(9) The CA15-3 measurement series are currently not recommended by American Society of Clinical Oncology (ASCO), European Society for Medical Oncology (ESMO), and National Comprehensive Cancer Network (NCCN) expert panel. However, this biomarker still exhibits moderate prognostic value in monitoring advanced stages of breast cancer, especially those related to metastasis. CA 15-3 is more reliable in the context of advanced breast cancer in the management of patient responses to systemic therapy.(21,22)

MiRNA-21 is an intron miRNA (the pri-miR-21 coding gene is in the intron region). The gene that codes for pri-miRNA-21 is on chromosome 17q23.2, overlapping with the gene that codes for the TMEM49 protein (VMP1).(23) MiRNA-21 plays a role in the process of cell proliferation, migration, invasion, preventing apoptosis of cancer cells.(15) The target gene is the tumor suppressor gene group. The miRNA-21 targets that have been identified are tropomyosin 1 tumor suppressor (TPM1), Signaling network of p53, PDCD4 and Maspin26.(24-26) MiRNA-21 can trigger the proliferation and transformation of breast cells through suppressing the translation of PDCD4 tumor suppressor protein, so that the role of PDCD4 in controlling neoplasm cell proliferation and transformation is decreased. (27) Due to the effect of miRNA-21 on its target, miRNA-21 can regulate cell proliferation, neoplastic transformation, and cell migration.(13)

Our research revealed that the miRNA-21 serum expression and CA 15-3 was found to be increased in breast cancer patients compared to healthy controls, however miRNA-21 showed a more vivid distinction with its specificity and sensitivity. These results were in agreement with the findings of previous study, which reported an increase in miRNA-21 in breast cancer patients compared with healthy group while CA 15-3 was elevated in patients with metastasis.(28)

\section{CA 15-3 Level}

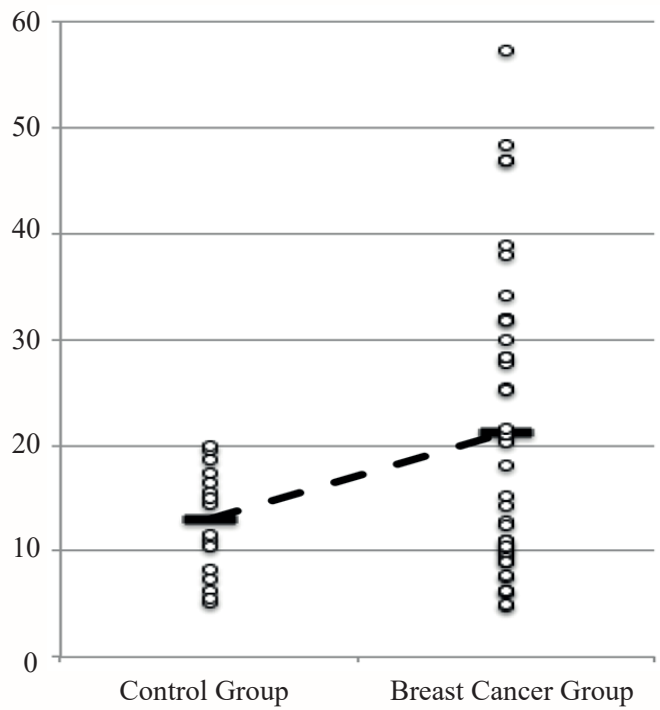

miRNA-21 Expression

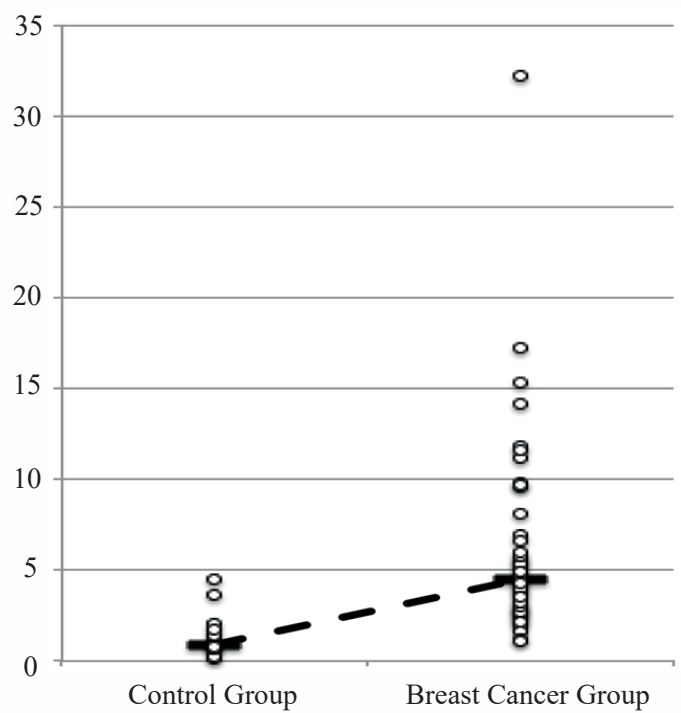

Figure 2. Comparison of CA 15-3 level and miRNA-21 expression in breast cancer and healthy control subjects. Black bar indicated median of CA 15-3 and miRNA-21 expression, white bullet indicated each sample data. Note: There were 8 outliers data ranging $>60$ (98.5; $130.2 ; 130.7 ; 428.3 ; 507 ; 564.1 ; 587.9 ; 3000)$ that excluded in the graphic of CA $15-3$ level. 
In healthy people, miRNA-21 is expressed lower than in the breast cancer group because there are no risk factors for breast cancer such as age, family history of cancer, hormonal (menarche status, estrogen hormone), diet, carcinogenic substances, genetic mutations do not arise and DNA damage on chromosome 17q23.1 location of miRNA-21 gene, so that biogenesis of miRNA-21 is running properly, and there is no over-expression of miRNA-21 that affects the process of cell growth, cell proliferation and differentiation and cell apoptosis.(29) We observed the low level of miRNA-21 in the healthy subjects in line with the literature study with a noticeable increase until reaching 4.92 folds increase in breast cancer subjects. The results of this study were inline with previous study, which reported 4.4 folds increase of miRNA-21 expression.(28) miRNA-21 expression levels might be able to differentiate early stage (stage 1 and 2) and advance stage breast cancer (stage 3 and 4). Similarly, previous study also reported increasing miRNA-21 expression in breast cancer along with the stage progression. $(30,31)$

The cut-off value of CA 15-3 that was obtained in the breast cancer group was 15.05 with an area under the ROC curve (ROC-AUC) of 0.701 (70.1\%, with $p=0.016)$ and sensitivity of $59.2 \%$ and specificity by $62.5 \%$. Meanwhile, the expression of circulating miRNA-21 in the breast cancer group exhibit 2.07 cut-off value with an area under the ROC curve (ROC-AUC) of 0.931 (93.1\%, with $p=0.000)$, and $91.8 \%$ sensitivity and $87.5 \%$ specificity. Although between CA 15-3 levels and miRNA-21 circulating expression obtained significant results, miRNA-21 has a wider and more meaningful AUC than CA 15-3. Moreover, the sensitivity and specificity of miRNA-21 are also higher than CA 15-3.

The results of this study are consistent with the previous studies which suggesting that the detection of circulating miRNA-21 level could serve as a novel superior biomarker for breast cancer, compared to CA 15-39,14,28. Therefore, the application of miRNA-21 as a breast cancer biomarker in the recent development could potentially provide clinician with better breast cancer biomarkers and ensuring better patients quality of live and management quality.

\section{Conclusion}

Although both CA 15-3 levels and miRNA-21 circulating expression showed a significant result between breast cancer group and control group, miRNA-21 expression increased consistently with breast cancer stage progression. miRNA-21 exhibit higher specificity and sensitivity with lower cut-off value. Therefore, miRNA-21 is superior breast cancer biomarker for Indonesian population. Yet, a multicenter study for evaluation of epigenetics factors which may affects miRNA-21 expression in Indonesia is still needed. Moreover, miRNA-21 may fill the gap that CA 15-3 still lacks in detecting breast cancer stage progression.

\section{References}

1. Bray F, Ferlay J, Soerjomataram I, Siegel RL, Torre LA, Jemal A Global cancer statistics 2018: GLOBOCAN estimates of incidence and mortality worldwide for 36 cancers in 185 countries. CA Cancer J Clin. 2018; 68: 394-424.

2. Forman D, Ferlay J, Steward BW, Wild CP. The global and regional burden of cancer. In: Stewart BW, Wild CP, editors. World Cancer Report 2014. Geneva: WHO Press; 2014.

3. Nassar FJ, Nasr R, Talhouk R. MicroRNAs as biomarkers for early breast cancer diagnosis, prognosis and therapy prediction. Pharmacol Ther. 2017; 172: 34-49.

4. Sandya Rani GSB, Bitla A. Breast cancer diagnosis - role of biomarkers. J Clin Sci Res. 2017; 6: 225-36.

5. Kharkwal S, Sameer A, Mukherjee A. Triple test in carcinoma breast. J Clin Diagnostic Res. 2014; 8: NC09-NC11.

6. Kahraman M, Röske A, Laufer T, Fehlmann T, Backes C, Kern F, et $a l$. MicroRNA in diagnosis and therapy monitoring of early-stage triple-negative breast cancer. Sci Rep. 2018; 8: 11584. doi: 10.1038/ s41598-018-29917-2.

7. Heywang-Köbrunner SH, Hacker A, Sedlacek S. Advantages and disadvantages of mammography screening. Breast Care. 2011; 6: 199-207.

8. Lauro S, Trasatti L, Bordin F, Lanzetta G, Bria E, Gelibter A, et al. Comparison of CEA, MCA, CA 15-3 and CA 27-29 in followup and monitoring therapeutic response in breast cancer patients. Anticancer Res. 1999; 19: 3511-5.

9. Gao J, Zhang Q, Xu J, Guo L, Li X. Clinical significance of serum miR-21 in breast cancer compared with CA153 and CEA. Chin J Cancer Res. 2013; 25: 743-8.

10. Shao Y, Sun X, He Y, Liu C, Liu H. Elevated levels of serum tumor markers CEA and CA15-3 are prognostic parameters for different molecular subtypes of breast cancer. PLoS One. 2015; 10: e0133830. doi: 10.1371/journal.pone.0133830.

11. Pissaia A, Bernard D, Scatton O, Soubrane O, Conti F, Calmus Y Significance of serum tumor markers carcinoembryonic antigen, CA 19-9, CA 125, and CA 15-3 in pre-orthotopic liver transplantation evaluation. Transplant Proc. 2009; 41: 682-4.

12. Heneghan HM, Miller N, Lowery AJ, Sweeney KJ, Kerin MJ. MicroRNAs as novel biomarkers for breast cancer. J Oncol. 2009; 2009: 950201. doi: 10.1155/2010/950201.

13. Pan X, Wang ZX, Wang R. MicroRNA-21: A novel therapeutic target in human cancer. Cancer Biol Ther. 2010; 10: 1224-32.

14. Mar-Aguilar F, Mendoza-Ramírez JA, Malagón-Santiago I, EspinoSilva PK, Santuario-Facio SK, Ruiz-Flores P, et al. Serum circulating microRNA profiling for identification of potential breast cancer biomarkers. Dis Markers. 2013; 34: 163-9.

15. Yan LX, Wu QN, Zhang Y, Li YY, Liao DZ, Hou JH, et al. Knockdown of miR-21 in human breast cancer cell lines inhibits proliferation, in 
vitro migration and in vivotumor growth. Breast Cancer Res. 2011; 13: R2. doi: 10.1186/bcr2803.

16. Qian B, Katsaros D, Lu L, Preti M, Durando A, Arisio R, et al. High miR-21 expression in breast cancer associated with poor diseasefree survival in early stage disease and high TGF- $\beta 1$. Breast Cancer Res Treat. 2009; 117: 131-40.

17. Fu SW, Chen L, Man YG. miRNA Biomarkers in Breast Cancer Detection and Management. J Cancer. 2011; 2: 116-22.

18. Suresh K, Chandrashekara S. Sample size estimation and power analysis for clinical research studies. J Hum Reprod Sci. 2012; 5: 7-13.

19. Uehara M, Kinoshita T, Hojo T, Akashi-Tanaka S, Iwamoto E, Fukutomi T. Long-term prognostic study of carcinoembryonic antigen (CEA) and carbohydrate antigen 15-3 (CA 15-3) in breast cancer. Int J Clin Oncol. 2008; 13: 447. doi: 10.1007/s10147-0080773-3.

20. Tiberio P, Callari M, Angeloni V, Daidone MG, Appierto V. Challenges in using circulating miRNAs as cancer biomarkers. Biomed Res Int. 2015; 2015: 731479. doi: 10.1155/2015/731479.

21. Mitchell PS, Parkin RK, Kroh EM, Fritz BR, Wyman SK, PogosovaAgadjanyan EL, et al. Circulating microRNAs as stable bloodbased markers for cancer detection. Proc Natl Acad Sci USA. 2008; 105: 10513-8.

22. Mirabelli P, Incoronato M. Usefulness of traditional serum biomarkers for management of breast cancer patients. Biomed Res Int. 2013; 2013: 685641. doi: 10.1155/2013/685641.

23. Ribas J, Ni X, Castanares M, Liu MM, Esopi D, Yegnasubramanian S, et al. A novel source for miR-21 expression through the alternative polyadenylation of VMP1 gene transcripts. Nucleic Acids Res. 2012; 40: 6821-33.

24. Feng YH, Tsao CJ. Emerging role of microRNA-21 in cancer (Review). Biomed Reports. 2016; 5: 395-402.

25. Ma X, Choudhury SN, Hua X, Dai Z, Li Y. Interaction of the oncogenic miR-21 microRNA and the p53 tumor suppressor pathway. Carcinogenesis. 2013; 34: 1216-23.

26. Zhu S, Wu H, Wu F, Nie D, Sheng S, Mo YY. MicroRNA-21 targets tumor suppressor genes in invasion and metastasis. Cell Res. 2008; 18: $350-9$.

27. Jiang LH, Ge MH, Hou XX, Cao J, Hu SS, Lu XX, et al. miR-21 regulates tumor progression through the miR-21-PDCD4-Stat3 pathway in human salivary adenoid cystic carcinoma. Lab Investig. 2015; 95: 1398-408.

28. Ibrahim H, Fattah A, Mahmoud NH, Elzoghby DM, Matar MM, Elshaer IMM. Clinical utility of circulating microRNA-21 in breast Cancer. Egypt J Hosp Med. 2018; 71: 2950-5.

29. Badr FM. Potential role of miR-21 in breast cancer diagnosis and therapy. Biotechnol Biomed Eng. 2016; 3: 1068.

30. Toraih EA, Mohammed EA, Farrag S, Ramsis N, Hosny S. Pilot study of serum microRNA-21 as a diagnostic and prognostic biomarker in Egyptian breast cancer Patients. Mol Diagn Ther. 2015; 19: 179-90.

31. Han JG, Jiang YD, Zhang CH, Yang YM, Pang D, Song YN, et al. A novel panel of serum miR-21/miR-155/miR-365 as a potential diagnostic biomarker for breast cancer. Ann Surg Treat Res. 2017; 92: 55-66. 